\section{A three-way chi-square program}

\author{
RICHARD J. STOKEY and JOHN D. WILLIAMS \\ University of North Dakota, Grand Forks, North Dakota 58201
}

While computer programs are widely available for a two-way chi-square analysis, such is not the case for third dimensional chi-square analyses. At least part of the reason for the lack of available computer solutions is the multiplicity of three-way solutions, depending upon the assumptions the researcher makes about the marginal frequencies. The present program uses what might be regarded as the simplest of all assumptions, that is, all cell frequencies are estimated from the marginal totals.

As an example, consider the following simple three-way solution: the three ways of classification are rows, columns, and groups (or slices). Forming two-way tables for groups, a $3 \times 2 \times 2$ situation can be shown:

\begin{tabular}{lrrr}
\hline & Column 1 & Column 2 & Totals \\
\hline & \multicolumn{2}{c}{ Group 1 } & \\
Row 1 & 12 & 8 & 20 \\
Row 2 & 6 & 24 & 30 \\
Row 3 & 12 & 58 & 70 \\
Totals & 30 & 90 & 120 \\
& & Group 2 & \\
Row 1 & 21 & 19 & 40 \\
Row 2 & 12 & 48 & 60 \\
Row 3 & 27 & 53 & 80 \\
Totals & 60 & 120 & 180 \\
\hline
\end{tabular}

The solution given in the present program is identical to the one described in Winer $(1971$, p. 858). Essentially, the problem is successively solved as a two-way chi-square analysis, disregarding groups when finding the rows by columns $x_{\mathrm{re}}^{2}$ value, disregarding columns when finding the rows by groups $x_{\text {rg }}^{2}$ value, and disregarding rows when finding the columns by groups $x_{c e}^{2}$ value. The three-way layout is then employed with the $x_{\text {reg }}^{2}$ value calculated as $x_{\text {Ieg }}^{2}=x_{\text {TOTAL }}^{2}-x_{\text {re }}^{2}-x_{\text {rg }}^{2}-x_{\text {cg }}^{2}$. The degrees of freedom for $x_{\text {reg }}^{2}$ are $(r-1)(c-1)(g-1)$. The total degrees of freedom are $(\mathrm{pqr}-1)-(\mathrm{p}-1)-(\mathrm{q}-1)-$ $(\mathrm{r}-1)$.

For the data in the sample program, $x_{\mathrm{rc}}^{2}=23.29, x_{\mathrm{rg}}^{2}=$ 5.56, $x_{\mathrm{cg}}^{2}=2.38, x_{\text {TOT AI }}^{2}=34.69$, and $x_{\mathrm{rcg}}^{2}=3.46$.

Description. This program performs a three-way chi-square analysis wherein no cell frequencies are fixed. Chi squares are formed for all two-way combinations and for the three-way layout.

Restrictions. The dimensions of the program are limited to a $12 \times 12 \times 12$ matrix. If necessary, the user can enlarge the dimensions; however, the printout may be somewhat more difficult to read.

Input. The problem allows the use of a free format. The first card includes the number of rows, columns, and groups, the numbers being separated by commas. The data cards follow in free format, the data being read in one group aa a time, each group being read in by rows. If the user prefers to use a fixed format, sufficient comment cards are included to allow this to occur. If a fixed format is used, then the first card is a control card containing the number of rows, columns, and groups in columns $1-5,6-10$, and 11-15 (right hand justified), respectively. The second card (for fixed format only) is a format card read in $\mathrm{F}$ format; the data cards follow.

Output. The data is output to correspond to (a) the three-way classification; (b) rows and columns, disregarding groups;(c) groups and columns, disregarding rows,; and (d) rows and groups, disregarding columns.

Following $b, c$, and $d$, the expected frequencies and $x^{2}$ values for this particular layout are shown. Then, the expected frequencies for the three-way classification are shown, with a $x^{2}$ value ( $x_{T}^{2}$ TOT $\left.A L\right)$ calculated on the three-way layout, $x_{\text {reg }}^{2}=$ $x_{\text {TOTAL }}^{2}-x_{\mathrm{rc}}^{2}-x_{\mathrm{rg}}^{2}-x_{\mathrm{cg}}^{2}$. A chi-square summary table is included with degrees of freedom and chi-square values for each of the four classifications. For the data given earlier, the printout is:

\begin{tabular}{lcr} 
& df & Chi-Square \\
\cline { 2 - 3 } Columns and Rows & 2 & 23.286 \\
Groups and Columns & 1 & 2.381 \\
Row and Groups & 2 & 5.556 \\
Rows, Groups, Columns & 2 & 3.460 \\
Total & 7 & 34.682
\end{tabular}

Computer and language. The program is written as a WATFIV program for the IBM $135 / 370$. Conversion to other FORTRAN programs are described in the comment cards. Conversions to other computer systems should be relatively simple.

Availability. For a free copy of the program with sample output, write either author at the Computer Center, The University of North Dakota, Grand Forks, North Dakota 58202.

\section{REFERENCES}

Winer, B. J. Statistical principles in experimental design.. New York: McGraw-Hill, 1971. 